\title{
La revolución digital en las unidades de información y la documentación
}

\author{
Francisco Javier García Marco \\ Universidad de Zaragoza
}

\subsection{Resumen}

Reflexiones sobre el impacto de la revolución digital en las unidades de infomación digital y de la actitud proactiva asumida por los profesionales e investigadores del área, ejemplificada a partir de la contextualización y consideración crítica del conjunto de estudios publicados en el número monográfico de la revista Scire (vol. 8, $\mathrm{n}^{\circ} .2$, julio-diciembre 2002) sobre La revolución digital en las bibliotecas y los centros de documentación. Se considera especialmente el futuro de la biblioteca en el entorno digital.

Palabras clave: Revolución de la información. Tendencias de investigación. Unidades de información y documentación. Bibliotecas digitales. Medios de comunicación de masas. Empresas.

\subsection{Abstract}

Reflections on the impact of the digital revolution in information and documentation units and on the proactive attitude taken by its community of professionals and researchers. The study is based on the critical appraisal and contextualization of the set of articles published in a monographic number of the journal Scire entitled The digital revolution in the libraries and documentation centres (Scire, vol. 8, n. 2, July-December 2002). The future of libraries in the digital context is specially considered.

Keywords: Information revolution. Research trends. Information and documentation units. Digital libraries. Mass media. Private firms. 


\section{Introducción}

El conjunto de estudios que se presentan en este segundo número del volumen octavo de la revista Scire es una muestra más del periodo floreciente que atraviesa en la actualidad la investigación en Biblioteconomía y Documentación. Esta vitalidad se aprecia tanto en la amplitud que están alcanzando las áreas de aplicación de los principios y técnicas de la Documentación como en la novedad de los temas que se vienen abordando en los últimos años. En cuanto al primer aspecto - las áreas aplicativas-, el presente número ofrece trabajos dentro del campo de las bibliotecas, la documentación periodística, la documentación empresarial y los archivos. Respecto a la novedad de los temas, cabe destacar la pasión con la que los investigadores de las Ciencias de la Documentación han acometido las implicaciones y posibilidades del nuevo entorno digital para el desarrollo de las labores de información y documentación. No es solo que estén abordando el imprescindible ajuste teórico y práctico a la nueva sociedad de la información —un enfoque pasivo o reactivo-, sino que la investigación se realiza con total compromiso tanto por parte de los colegas más jóvenes — que simbolizan el empuje y las nuevas fronteras - como por parte de aquéllos con una dilatada trayectoria profesional que les convierte en adalides de la ampliud de visión, la experiencia y el rigor.

El conjunto de estudios que se presentan al lector tiene, pues, como tema de fondo y preocupación fundamental el impacto de la revolución digital en las unidades de información y documentación. A continuación se presentarán los aspectos que nos han parecido más destacables de los diferentes capítulos, y, a tenor de esa reflexión crítica, se profundizará en algunos temas, especialmente, en el del futuro de la biblioteca en el entorno digital.

\section{El futuro de la profesión, de la educación y de la teoría bibliotecaria}

Abren el número dos interesantísimos estudios sobre el futuro de la profesión, la educación y la teoría bibliotecológica-documentológica a cargo de dos colegas brasileños y uno cubano, que sitúan dicho futuro en el marco del concepto de gestión de la información. En cierta manera, la interacción entre profesión, educación, investigación y construcción teórica constituye el motor de cuatro tiempos que anima el desarrollo de las Ciencias de la Documentación, como ocurre, por otra parte, en la gran mayoría de las ciencias aplicadas. Los dos trabajos que presentamos a continuación se caracterizan por la profunda unión entre esos cuatro aspectos: investigación para la educación, educación a través de la investigación, educación desde la profesión, educación para la profesión... De alguna manera abordan la complejidad de las interacciones entre estos conceptos fundamentales con la reversibilidad y potencialidad combinatoria que Piaget otorga al estadio del pensamiento formal, y muestran que es necesario pensar, sentir y actuar desde los cuatro puntos, aunque el énfasis de nuestro trabajo profesional se sitúe en uno de ellas.

Scire. $8: 2$ (jul.-dic. 2002) 9-21. 
El artículo de Edna Lucía da Silva y de Miriam Vieira da Cunha (2002) aborda el encuadre de la educación del profesional de la información y de la documentación a partir de los retos del momento histórico presente, caracterizado por la revolución de la información y la globalización. Su trabajo destaca por su profunda orientación a los valores, más allá de los desarrollos infraestructurales que caracterizan el momento actual, a los que, por otra parte, prestan cuidadosa atención y a los cuales valoran de forma positiva. El artículo comienza con un detallado análisis - a partir de las ideas de Morin (2001, 2002; Morin, Roger y Motta, 2002) - entorno a los principios-faro propuestos por la Unesco para la educación en el siglo XXI: aprender a conocer, aprender a vivir juntos, aprender a hacer y aprender a ser. Se trata de una concepción profundamente humanista que sitúa las tecnologías como una parte fundamental de la formación —un "aliado imprescindible", al decir de las autoras-, pero siempre al servicio de valores superiores, orientados a la consecución de la felicidad de todos. Desde este punto de vista, la educación en general y la educación universitaria en particular requieren una transmisión crítica e implicada del conocimiento orientada a la consecución de la madurez personal y la convivencia responsable. Fijado el marco filosófico del proceso educativo, nuestras colegas brasileñas abordan la misión del profesional de la información del futuro y, en definitiva, el que debe ser el marco específico de la formación de los bibliotecarios y documentalistas en los años venideros. Acertadamente, sitúan el énfasis de la actividad profesional y de las necesidades de formación entorno al problema de la sobreabundancia de la información, cada vez más agudo, que deberá ser abordado desde el uso inteligente de las nuevas tecnologías.

Por su parte, el artículo de Emilio Setién (2002) considera el futuro de la teoría bibliotecológica en el siglo XXI, tal y como se vislumbra desde la experimentada y reflexiva trayectoria de este investigador cubano. Abre su estudio un cuidadoso análisis del recorrido de la teoría bibliotecológica durante el siglo XX, hasta desembocar en los nuevos paradigmas de la gestión de la información y, ahora, del conocimiento. Emilio Setién concibe la gestión de la información sobre todo como estructuración y organización de la información, y sitúa la explicitación de las relaciones entre los contenidos como parte fundamental de lo que él entiende como la "labor creativa" del bibliotecario, íntimamente unida, en su vertiente de diseminación de la información, a las labores de animación de la lectura, entendida en un sentido amplio. Esta visión de Emilio Setién conecta ¿sorprendentemente? con aquella nueva profesión vislumbrada por Vannevar Bush (1945) que consistiría en crear caminos de lectura a través del mundo de los documentos conectados mediante el hipertexto. Ciertamente, desde la perspectiva que otorga una amplia formación humanista y filosófica y el compromiso sostenido por el desarrollo social, el profesional de la información colabora — junto con el educa-

Scire. $8: 2$ (jul.-dic. 2002) 9-21. 
dor y el informador, y con el resto de los profesionales cuando asumen estas funciones- en la introducción de las personas en el mundo de la cultura, proponiendo caminos de lectura y "visualización" de acuerdo con los valores, necesidades y metas de la comunidad concreta con la que se han comprometido. Volviendo a sus propuestas específicas, los hitos del camino que Emilio Setién propone para Cuba pueden servir también para orientar los programas bibliotecológicos de cualquiera de nuestros países, tanto en sus aspectos profesionales como, consecuentemente, académicos: a) investigar la relaciones entre la función bibliotecaria y su entorno; b) actualizar la formación profesional de acuerdo al punto anterior; c) estudiar el papel de las bibliotecas en la gestión del conocimiento y de sus resultados; d) atender tanto a la creación de colecciones reales como al acceso a fuentes remotas y virtuales, sin descuidar la preservación de la información; e) facilitar "la educación popular para el empleo de los medios tecnológicos en la búsqueda informativa, y al fortalecimiento de hábitos de lectura que sustenten y hagan que el fenómeno bibliotecario se desenvuelva de forma racional, rentable y efectiva."

\section{La urgencia de la biblioteca digital: entre la realidad y la ficción}

El siguiente conjunto de estudios que animan estas páginas está dedicado a uno de los énfasis fundamentales de nuestro momento presente: la biblioteca digital. Efectivamente, la biblioteca digital constituye el artefacto fundamental entorno al cual se está reelaborando la teoría bibliotecológica para la sociedad de la información. La biblioteca digital se hace posible precisamente gracias a la revolución de las tecnologías de la información y la documentación, y se nutre de las mismas ambiciones holísticas respecto al mundo de la información digital que las que tenía la biblioteca universal respecto al mundo del conocimiento escrito. En esa ambición, la biblioteca digital se debate entre los polos de la realidad y la imaginación, del pasado y del futuro, constituyéndose, a pesar de sus contradicciones y promesas exageradas, en la indiscutible punta de lanza del futuro.

Consideramos, sin embargo, que la biblioteca digital triunfará —y de hecho está triunfando- en la medida en que deje de ser un objeto casi platónico para comprometerse con las comunidades de usuarios y profesionales concretos, asumiendo los estándares rigurosos de las bibliotecas tradicionales y apoyándose en los grandes principios de la ciencia bibliotecaria - acceso público universal, normalización y cooperación bibliotecaria-, plenamente compartidos desde otros puntos de vista por los principales actores de la Internet —el World Wide Web Consortium y la Internet Society-. De esta forma, veremos surgir, casi sin sentirlo, ladrillo a ladrillo, hilo a hilo y bit a bit, a través de la colaboración de los distintos puntos de la red que forma su embrión, la "biblioteca digital univesal" que, sin duda, va a conformar uno de los grandes monumentos que el siglo XXI va a legar a la Humanidad.

Scire. $8: 2$ (jul.-dic. 2002) 9-21. 
La biblioteca digital que se vislumbra en la actualidad puede que pronto pierda el mote para quedar en biblioteca, porque lo digital será obvio y constituirá la columna vertebral - hegemónica, pero nunca exclusiva ni excluyente- de los servicios bibliotecarios. Por una parte, los documentos digitales y el acceso a los mismos se habrán extendido a lo largo de todas las redes bibliotecarias, y la biblioteca digital se comprenderá — más allá de los sueños de virtualidad- como tarea de organizaciones humanas, con un anclaje profundo en el mundo real, aunque, sin duda, afectadas por las posibilidades de trabajo distribuido y de audiencia global que ofrece la red. La biblioteca y la Biblioteconomía deberán seguir ocupándose de todos los documentos, sea cual sea su soporte o formato, aunque la idea de documento multimedia le proporcionará unidad de concepto y el acceso digital constituirá una prioridad al servicio de la accesibilidad universal.

La biblioteca virtual — carente en teoría de infraestructura física, central o distribuida - se puede concebir, quizá, como un trasunto o como una extensión al servicio de los usuarios remotos de la biblioteca total —o sencillamente y de nuevo biblioteca - hacia la que caminamos. Pero la biblioteca virtual no debería ser la única forma de biblioteca, sino tan solo uno de sus interfaces. En este aspecto como en otros, nos enfrentamos al sueño pintoresco de la escuela sin profesores, la medicina sin médicos, la administración sin funcionarios o la banca sin atención personalizada... distintas formas del mito de una sociedad sin personas, cuyos límites —una vez pasado el entusiasmo inicial, sin duda, comprensible dadas las implicaciones revolucionarias de los cambios que ha sufrido nuestra sociedad entorno al cambio de siglo- resultan obvios y han requerido las necesarias correcciones. En definitiva, en el resto de las actividades humanas lo digital se va descubriendo como una extensión de las actividades de instituciones bien ancladas en el mundo real, y la biblioteca no tendría por qué ser una excepción a esta regla general.

Sin embargo, la seguridad en la permanencia de la institución no puede extrapolarse a la supervivencia de las bibliotecas concretas en su forma actual. La realidad nos muestra la profundidad del impacto de los cambios en la organización bibliotecaria y la estructura de la biblioteca: colecciones de revistas y obras de referencia accesibles en línea desde distribuidores especializados, delegación de las labores de catalogación en manos de los proveedores de documentos, publicación digital de los fondos propios únicos o raros a través de consorcios localizados fuera del centro de cálculo de las bibliotecas, competencia entre las bibliotecas especializadas a través de la red haciendo obsoletos catálogos y servicios locales, y un largo etcétera.

Y es que la indiscutible función que desempeñan las bibliotecas y los centros de información y documentación no se corporeiza necesariamente en una forma concreta de validez universal. La función no hace unilateralmente la forma. Por

Scire. $8: 2$ (jul.-dic. 2002) 9-21. 
el contrario, la forma nace de la interacción entre las posibilidades funcionales del organismo concreto, de su patrimonio genético — variable, polivalente, mutante y recombinable- y del contexto; y lo mismo ocurre en nuestro campo de desarrollo profesional y científico.

De hecho, una mirada desapasionada muestra como el entorno digital se rige también - y entre otras leyes - por el principio de la supervivencia de los más adaptados, y que detrás de las "bibliotecas digitales" más potentes existen instituciones fuertes y flexibles con una sólida organización central y una amplia red de sucursales - o colaboradores en el caso de los proyectos cooperativos- y alianzas. Respecto a ellas, la biblioteca digital funciona como una extensión capaz de ampliar enormemente su radio de acción y su red de usuarios o —en muchas ocasiones - incluso clientes, hasta alcanzar una audiencia prácticamente mundial. Los casos de servicios como Eric de la National Library of Education de los Estados Unidos, Medline de la National Library of Medicine de los Estados Unidos o los proporcionados por el instituto de información artística del Paul Getty Trust constituyen un buen ejemplo de ello. Por otro lado, también hay que destacar hasta qué punto las bibliotecas se están conviertiendo en puntos de consumo de los servicios ofrecidos por las grandes compañías de distribución editorial, que pueden realizar bajo demanda todas las actividades de creación y mantenimiento de colecciones que tradicionalmente se ejecutaban in situ. La vertiente positiva de esta evolución es que la fuerza de trabajo de la biblioteca y el centro de documentación puede dirigirse cada vez más hacia la interacción directa con el usuario específico. Pero, en fin, dejemos las reflexiones de carácter general para abordar la presentación de los trabajos que se publican entorno a los problemas de la biblioteca digital, cuyos autores han profundizado en los aspectos que se han abordado hasta aquí a manera de introducción.

El primer capítulo de la serie corre a cargo de Blanca Rodríguez Bravo y Lourdes Santos de Paz (2002), que proporcionan una visión general del advenimiento de la biblioteca digital y de la su evolución en el último cuarto del siglo XX a través de la discusión de sus diferentes denominaciones. Las autores explican la biblioteca digital a partir de la aparición, desarrollo y generalización de los documentos digitales. Discuten el concepto de "biblioteca híbrida" como objetivo de los esfuerzos actuales - que deben asegurar tanto el acceso a los documentos "tradicionales", como su digitalización para el acceso remoto y el acceso a documentos digitales externos-, y se hacen eco de la discusión entre los que opinan que ésta constituye un estadio transicional hacia la biblioteca virtual y los que ven en ella el estado natural, previsible y deseable de la biblioteca del futuro. Su posición está en cualquier caso fundamentada en principios: "Consideramos que sería conveniente que las bibliotecas híbridas o digitales, integradoras de todo tipo de soportes y servicios, convivan con otras virtuales que dediquen sus

Scire. $8: 2$ (jul.-dic. 2002) 9-21. 
presupuestos a la digitalización masiva de documentos analógicos y permitan acceder a los contenidos a los usuarios que viven alejados de los núcleos de población principales. A comienzos del siglo XXI, y con las facilidades que Internet proporciona, no se puede seguir fomentando la existencia de ciudadanos de primera y de segunda, informativamente hablando." (Rodríguez y Santos, 2002, p. 48).

María del Carmen Negrete (2002), especialista mexicana en el desarrollo de colecciones para bibliotecas universitarias y especializadas, analiza un tema de la máxima actualidad para la biblioteca del cambio de siglo: la selección de recursos digitales. Se trata de un análisis sintético, centrado, sensato y práctico sobre esta cuestión, que considera cuidadosamente el estado de la cuestión y la literatura norteamericana sobre el tema. El análisis de Carmen Negrete es netamente bibliotecológico, no tecnocéntrico, y plantea la selección de los recursos digitales desde la misión y objetivos de la biblioteca, el análisis estratégico de la colección, la consideración de sus usuarios, oferta y recursos, y la cuidadosa planificación y evaluación del proceso en su conjunto. Destaca su modelo de selección, en el que considera con detalle aspectos relacionados con el contenido, la autoría, actualidad, facilidad de uso, presentación, interactividad, adecuación, el coste y los medios requeridos. Finalmente, en la línea de lo que venimos discutiendo sobre la validez de los principios generales de la Bibliotecología en el nuevo contexto digital, Carmen Negrete concluye que los principios de la selección no cambian respecto a los medios impresos, aunque sí se deben ajustar los métodos, los criterios y las prácticas específicas.

Por su parte, Lourdes Fernández Ramírez, Alfredo Sánchez y Alberto García (2002) plantean un ejemplo de las posibilidades que las colecciones digitales ofrecen para hacer accesibles en colaboración con los autores documentos muy valiosos, pero que no presentan una buena relación coste/beneficio para su distribución por los canales editoriales habituales. Se trata de la presentación del proyecto de creación de una colección digital de tesis en el marco del marcoproyecto University Digital Libraries for All (U-DL-A) de la Biblioteca Universitaria de La Universidad de las Américas. En este caso sí que estamos en presencia de un proyecto orientado a la "virtualidad", pues busca digitalizar todo el proceso de creación, producción, evaluación, publicación y difusión de tesis de maestría y doctorado aprovechando al máximo las posibilidades del hipertexto distribuido para la gestión y difusión de información científico-técnica. Sin embargo, los autores anotan cuidadosamente que la biblioteca digital de tesis tiene su sitio en la biblioteca digital, y ésta, a su vez, en la biblioteca universitaria de la Universidad de Las Américas, mostrando de manera fehaciente la imbricación de los proyectos digitales y aún virtuales en el marco de la institución bibliotecaria.

Scire. $8: 2$ (jul.-dic. 2002) 9-21. 
Manuel José Pedraza (2002) aborda otro tema muy especializado dentro de la Bibliotecología y las Ciencias de la Documentación en General, pero también de extraordinario interés: el libro antiguo en la red. No se centra tan solo en la publicación electrónica de las colecciones de libros antiguos de las bibliotecas, sino que explora igualmente los recursos dedicados a la investigación en libro antiguo e historia del libro, los recursos para el tratamiento del material antiguo y las páginas web de libreros anticuarios y bibliófilos. Con humor, señala en su título que se ocupa del "contacto de los extremos" — esto es, del libro antiguo, por un lado, y de la Internet, por el otro-, situación que nos muestra hasta qué punto la sociedad de la información ha calado en todos los aspectos de la vida moderna, y, lo que quizá es aún más interesante, cómo ámbitos muy especializados en los que la información fluía con dificultad se han visto extraordinariamente beneficiados por las nuevas tecnologías de la información y la comunicación. Además, el cuidadoso análisis de Manuel Pedraza muestra también las debilidades de la información en Internet; en particular, el contraste que existe entre la gran cantidad de recursos disponibles frente a su redundancia y, en la mayoría de las ocasiones, escasa calidad. Por último, muestra también, en la línea del panorama que venimos, como son las grandes instituciones - a través de sus catálogos y servicios de valor añadido- - y el sector comercial los agentes que más están empujando el desarrollo de la información sobre el libro antiguo en Internet.

Seguidamente, Francisco Javier García Gómez y Antonio Díaz Grau (2002) presentan un cuidadoso análisis sobre el desarrollo de los sistemas de información en la World Wide Web de las tres bibliotecas de la Región de Murcia que en el momento del estudio contaban con ellos. Su trabajo permite observar con detalle los servicios ofrecidos por estas bibliotecas y ofrece una metodología de análisis.

Finalmente, cierra la parte dedicada a las bibliotecas digitales el interesantísimo estudio de Claudia Paz Yanes (2002). Esta bibliotecaria nos ofrece una perspectiva de la historia de las bibliotecas y los bibliotecarios desde un punto de vista poco común: la visión que la ficción presenta del mundo del libro y las bibliotecas. El estudio de Claudia Paz se centra especialmente en el séptimo arte partiendo de las primeras películas hasta llegar a los géneros contemporáneos. Además, contrasta las conclusiones extraídas del cine con diversos ejemplos extraídos de la literatura y el comic. La autora - como sus lectores y otros estudiosos que se han ocupado del tema- sale fascinada de ese recorrido por el imaginario público de la profesión — que nos pone en contacto con nuestras raíces y también con nuestros demonios- y termina su contribución proponiendo múltiples vías de estudio dentro de un área de investigación crítica para comprender tanto la historia de la biblioteca y los bibliotecólogos como su impacto en el desarrollo de la civilización y, recientemente, de la sociedad de la información. 


\section{Las unidades de información y documentación en los medios de comunicación de masas}

Indudablemente, uno de los campos de aplicación más importantes, consolidados e influyentes dentro de la información y documentación es el que configuran los medios de comunicación de masas.

El artículo que abre esta sección del volumen — a cargo de María Àngels Jiménez, María Eulàlia Fuentes y Alfons González (2002) de la Universidad Autónoma de Barcelona (UAB) — estudia las nuevas posibilidades que ofrecen la ediciones digitales de los medios de comunicación a los documentalistas y, muy en particular, las nuevas oportunidades que plantean los denominados servicios de valor añadido (SVA) de prensa. Se trata de un trabajo pionero del grupo de investigación de la UAB que muestra los frutos que la metodología de análisis documental puede dar en la mejora de los productos digitales. En este sentido, establecen un conjunto de principios de actuación desde un punto de vista documental orientados a la mejora cualitativa de dichos SVA. Por otra parte, el cuadro de principios directores que nos ofrecen constituye una estupenda guía para la evaluación de nuestros servicios y productos en general: multidisciplinariedad, planificación, utilidad, universalidad, inteligibilidad, rigor, recuperabilidad, pesonalización y actualización. María Eulalia Fuentes y los investigadores de la UAB son un excelente ejemplo de la rápida capacidad de reacción de los equipos de investigación y aplicación de nuestra área para acometer nuevos problemas e implicarse en la vanguardia de la sociedad de la información.

El capítulo de Maria de Fátima Garbelini (2002), de la Universidade Federal de Goiás (Brasil), sobre los nuevos retos y tareas profesionales para el documentalista y la gestión de la información en los medios de comunicación, anclado profundamente en su experiencia, proporciona diversas lecciones en las mismas líneas que venimos tratando,. En particular insiste en la necesidad de que el documentalista de medios se implique en equipos interdisciplinares, actúe desde el compromiso social y con rigor, y reciba una formación especializada en periodismo y en el abordaje de los nuevos documentos audiovisuales y multimedia, etc. Fatima Garbelini termina su trabajo con una cita de Bernardino J. Cebrián Enrique (1998), que sintetiza de alguna manera la necesidad de un cambio de paradigma en la relación entre los profesionales de la documentación y de la comunicación - susceptible de ser aplicada a las relaciones con otros profesionales especializados-: "Los periodistas tienen que reconocer que necesitan documentar la información. Los profesionales de Documentación tienen que acercarse más a los periodistas, e incluso pensar y actuar como ellos." Ese cambio de paradigma está ciertamente en curso y pasa porque bibliotecarios y documentalistas, de un lado, y especialistas temáticos — periodistas, docentes, etc.—, del otro, dejen de considerarse usuarios o ayudantes, y empiecen a reconocerse como profesionales colegas

Scire. $8: 2$ (jul.-dic. 2002) 9-21. 
al servicio de la misión de sus organizaciones, que trabajan en equipo para la consecución de objetivos específicos. Parece claro que es necesario seguir profundizando en este camino y derribar barreras innecesarias y prejuicios demasiado arraigados. Para eso, como muy bien señalan estos autores para el campo específico del periodismo, debe actuarse en las dos direcciones: por un lado, enseñar documentación en las diferentes carreras; por el otro, los documentalistas deben recibir una formación específica en el campo científico o técnico que habrá de constituir su especialidad. El movimiento actualmente en curso de reforma del Espacio Europeo de Educación Superior - que concede especial atención a la formación de posgrado y a las dobles enseñanzas- debería aprovecharse para especializar el trabajo de las diferentes universidades, reagrupando sus docentes en el marco de los convenios pertinentes, usando como mencanismos las nuevas tecnologías y los intercambios docentes. Solo así se podrán crear los suficientes equipos especializados que se ocupen con solvencia del elevado número de programas específicos que serían necesarios.

Finalmente, cierra este apartado dedicado a la documentación de medios un trabajo en el que María Ángeles López Hernández y María Dolores Rodríguez Brito presentan el estado actual de un proyecto desarrollado por el Grupo de Investigación en Comunicación Científica y Desarrollo del Departamento de Periodismo de la Universidad de Sevilla, que se ha ocupado de analizar la situación real de la práctica documental en las empresas audiovisuales de la Comunidad Autónoma de Andalucía. Su trabajo tiene la virtud de poner el contrapunto al panorama optimista que presentan el resto de los artículos, mostrando, por un lado, las limitaciones de especialización, tecnología, normalización y acceso en las que se desenvuelve el trabajo documental en las empresas locales privadas, y, por el otro —y más allá de una simple lectura "desalentadora"—, la importante y apasionante labor que queda por delante.

\section{Las unidades de información y documentación en el sector empresarial}

En la recta final del volumen presentamos dos trabajos especializados en información y documentación empresarial, uno de ellos a cargo del documentalista zaragozano Miguel Ángel del Prado Martínez — que aborda el diseño e implementación de los servicios de documentación en las organizaciones empresariales - y otro de la profesora de Ciencias Económicas y Empresariales de la Universidad de León María Rosa Llamas Alonso - que analiza los retos que, para la investigación en marketing internacional, plantea la información en Internet.

Frente a los que creen que la Archivística, la Biblioteconomía y la Documentación deben ser disciplinas estancas, el trabajo de Miguel Ángel del 
Prado muestra, sin pretenderlo explícitamente, como un profesional de la información y la documentación puede planificar y dirigir — desde el concepto de gestión de la información y del conocimiento- una unidad de información integrada en la que funcionen conjuntamente la biblioteca, el centro de documentación y el archivo, compartiendo plataformas informáticas y servicios de difusión y alerta de información. No se trata de confundir especialidades y servicios, ni de pensar que la integración tiene que ser siempre la única y mejor solución, ni, mucho menos, de establecer recetas centralistas para la coordinación de las funciones; pero sí resulta necesario mostrar los beneficios de una gestión integral de la información al servicio de la misión y los objetivos de las organizaciones ayudada de las enormes posibilidades que abren las infraestructuras informáticas y telemáticas. Sin duda, el proyecto de Miguel Ángel del Prado y de las organizaciones que lo han demandado y apoyado - Federación de Empresarios del Metal de Zaragoza (FEMZ), Confederación de la Pequeña y Mediana Empresa Aragonesa (CEPYME Aragón), Confederación de la Pequeña y Mediana Empresa de Zaragoza (CEPYME Zaragoza) y Fundación "Cultura y Empresa"son un excelente ejemplo de ello, llevado a cabo con notaria eficacia a pesar de las limitaciones de personal, de las que los pequeños y medianos centros de información y documentación suelen adolecer.

Por su parte, el trabajo de Rosa Llamas muestra desde la sensibilidad de una experta temática la importancia de los sistemas de información y documentación, en este caso desde el campo del comercio exterior. A partir de la constatación del papel fundamental que juegan las fuentes de información sobre comercio exterior en Internet para hacer frente a los retos de la globalización económica y empresarial y de la necesidad de organizar los datos procedentes de las diversas fuentes, Rosa Llamas presenta el Sistema de Información de Mercados Exteriores integrado en el que su equipo está trabajando. Interesa destacar — como lección para los profesionales de la información y la documentación- la existencia de un lenguaje común y de unos principios compartidos entorno a los problemas de la información, las necesidades informativas, y los sistemas de información. Este lenguaje común refleja como las Ciencias de la Documentación comparten con otras disciplinas el paradigma informacional y se encuadran conceptual y metodológicamente dentro del amplio abanico de las ciencias sociales aplicadas.

\section{Los archivos en la era de la información}

No podía faltar en esta monografía algún trabajo relacionado con los archivos, en esta ocasión a cargo de Luis Lluch Baixauli, Carmen María Brugarolas Ros y María Martín Hernández. Los autores abordan también un tema fronterizo dentro de la Archivística, en este caso la descripción de fondos fotográficos, tarea que abordan con los últimos útiles normativos y conceptuales de esta ciencia, señala-

Scire. $8: 2$ (jul.-dic. 2002) 9-21. 
damente, la norma ISAD (G), cuyo uso ilustran con algunos ejemplos. Efectivamente, la comunidad archivística - junto con el resto de los profesionales informativo-documentales - se ve afectada y responde proactivamente a los retos de la sociedad de la información, fundamentalmente a aquéllos planteados por la creciente globalización, las redes distribuidas, los documentos digitales y las posibilidades y problemas planteados por los sistemas de información en su conjunto.

\section{Conclusión}

A través de los trabajos presentado en la presente monografía, se aprecia como los profesionales, docentes, investigadores y teóricos de las ciencias de la Documentación están demostrando una gran vitalidad en acometer los retos del impacto de la revolución digital en todas sus especialidades de trabajo: bibliotecas, centros de documentación, archivos y servicios de información.

Pero no solo se limitan a reaccionar y a adaptarse. Aprovechando los nuevos vientos, están abriendo campos nuevos en actividades muy diversas, de las que la publicación en línea de las colecciones digitales de tesis o la mejora de los denominados servicios de valor añadido de la prensa digital pueden servir como ejemplo. Y están utilizando ese impulso para posicionarse mejor si cabe en el corazón de los equipos interdisciplinares que dirigen las instituciones y las empresas, tomando una posición activa y adquiriendo una visión global y estratégica.

En el nombre del equipo de editores, quiero manifestar nuestro orgullo por haber podido participar a través de este volumen en la difusión de sus trabajos, de sus esfuerzos y —ipor qué no decirlo claramente?— de sus éxitos.

\section{Referencias}

Bush, V. As We May Think. // Atlantic Mounthly. 176 (July 1945), 101-108.

Cebrián Enrique, Bernardino J. (1998). Nuevos enfoques sobre la misión del documentalista en los medios de comunicación.// Documentación de las Ciencias de la Información. 21 (1998) 91-100.

Fernández, Lourdes; Sánchez, Alfredo; García, Alberto (2002). TALES: Integración de tesis en una biblioteca digital avanzada. // Scire. $8: 2$ (jul.-dic. 2002) 61-70.

Garbelini, Maria de Fátima (2002). El trabajo del documentalista y la gestión de la información en los medios de comunicación: nuevos retos y tareas profesionales. // Scire. $8: 2$ (jul.-dic. 2002) 153-158.

García Gómez, Francisco Javier; Díaz Grau, Antonio (2002). Los sistemas de información web en las bibliotecas públicas: la situación en la región de Murcia. // Scire. 8 : 2 (jul.-dic. 2002) 91-116.

Jiménez, María Àngels; Fuentes, Eulalia; González, Alfons (2002). El profesional de la información ante el reto de los medios de comunicación digitales: el caso de la prensa. // Scire. $8: 2$ (jul.-dic. 2002) 141-152. 
Llamas Alonso, María Rosa (2002). La información en internet: nuevos retos para la investigación de marketing internacional. // Scire. 8 : 2 (jul.-dic. 2002) 189-100.

Lluch, Luis; Brugarolas, Carmen; Hernández, María Martín (2002). El archivo municipal de San Javier: propuesta de descripción del fondo fotográfico. // Scire. $8: 2$ (jul.-dic. 2002) 201-210.

López Hernández, María Ángeles; Rodríguez Brito, María Dolores (2002). La documentación audiovisual en las empresas audiovisuales locales e instituciones universitarias andaluzas. // Scire. $8: 2$ (jul.-dic. 2002) 159-172.

Morin, Edgar (2001). Los siete saberes necesarios para la educación del futuro / [traducción de Mercedes Vallejo-Gómez, con la contribución de Nelson Vallejo-Gómez y Françoise Girard]. Barcelona : Paidós Ibérica , [2001].

Morin, Edgar (2002). La mente bien ordenada: repensar la reforma, reformar el pensamiento. Barcelona : Seix Barral, 2000.

Morin, Edgar; Roger Ciurana, Emilio; Domingo Motta, Raúl (2002). Educar en la era planetaria: el pensamiento complejo como "método" de aprendizaje en el error y la incertidumbre humana / elaborado para la UNESCO por Edgar Morin, Emilio Roger Ciurana, Raúl Domingo Motta. [Valladolid] : Secretariado de Publicaciones e Intercambio Editorial, Universidad de Valladolid , [2002].

Negrete Gutiérrez, María del Carmen (2002). Criterios de selección para recursos digitales. // Scire. $8: 2$ (jul.-dic. 2002) 53-60.

Paz Yanes, Claudia (2002). Bibliotecas de cine: una revisión de la imagen de las bibliotecas y los bibliotecarios en el séptimo arte. // Scire. 8 : 2 (jul.-dic. 2002) 117-140.

Pedraza Gracia, Manuel José (2002). El libro antiguo en la red o El contacto de los extremos. // Scire. $8: 2$ (jul.-dic. 2002) 71-90.

Prado Martínez, Miguel Ángel del (2002). El servicio de documentación en las organizaciones empresariales. // Scire. $8: 2$ (jul.-dic. 2002) 173-188.

Rodríguez Bravo, Blanca; Santos, Lourdes (2002). Del documento digital a la biblioteca virtual. // Scire. $8: 2$ (jul.-dic. 2002) 43-52.

Setién, Quesada Emilio (2002). Posibles derroteros de la teoría bibliotecológica en el siglo XXI. // Scire. 8 : 2 (jul.-dic. 2002) 33-42.

Silva, Edna Lucia da; Cunha, Miriam Vieira da (2002). Los profesionales de la información y los dilemas educacionales del mundo globalizado. // Scire. $8: 2$ (jul.-dic. 2002) 23-32. 